\title{
Reversed offsetting of the septal attachments of the atrioventricular valves and Ebstein's malformation the morphologically mitral valve
}

\author{
MAURICE LEUNG, MICHAEL L RIGBY, * ROBERT H ANDERSON, * \\ RICHARD K H WYSE, $\dagger$ FERGUS J MACARTNEY†
}

From the ${ }^{\star}$ Brompton Hospital and $\nmid$ Hospital for Sick Children, London

SUMMARY Ebstein's anomaly of the tricuspid valve is characterised by downstream displacemerit of the tricuspid leaflets. In the case of the septal leaflet, the result is exaggerated offsetting of the atrioventricular valves. Thus it might be supposed that in Ebstein's anomaly of the mitral valve, downstream displacement of the aortic (septal) leaflet of the mitral valve could move its attachs ment beyond that of the tricuspid valve, resulting in reversed offsetting. To examine this hypothe esis three patients each with a concordant atrioventricular connection were studied. The firs with Marfan's syndrome and without an abnormal mitral valve, had reversed offsetting shown by echocardiography. The second, without Marfan's syndrome and with Ebstein's anomaly of the mitral valve, showed echocardiographically normal offsetting but a displaced mural leaflet. The third, in whom Ebstein's anomaly of the mitral valve was confirmed at necropsy, showed a田 the features of the second case, with normal offsetting of the atrioventricular valves.

Thus reversed offsetting is not a feature of Ebstein's anomaly of the mitral valve and in the fir塱 case this feature was caused by upward displacement of the tricuspid valve secondary to dilatio of the aortic root.

In hearts with the usual atrial arrangement (solitus) and concordant atrioventricular connection, four chamber echocardiographic sections show that the septal attachment of the mitral valve is usually closer to the atria than the septal attachment of the tricuspid valve. The detection of mirror image arrangement of this anticipated offsetting of the atrioventricular valves in hearts with the usual atrial arrangement has, when the septal structures are intact, been described as a significant finding in some hearts with discordant atrioventricular connection. ${ }^{1-3}$ We have encountered this reversal of the normal offsetting of the atrioventricular valves in the presence of concordant atrioventricular and ventriculoarterial connections with the usual atrial arrangement. This suggested to us the diagnosis of Ebstein's anomaly of the left sided mitral valve, a rare but well recognised lesion. ${ }^{45}$ Subsequent examination of a necropsy example of this

Requests for reprints to Professor Robert H Anderson, Department of Paediatrics, Cardiothoracic Institute, Brompton Hospital, Fulham Road, London SW3 6HP.

Accepted for publication 15 September 1986 malformation together with a case examin echocardiographically showed that this lesion does not produce reversed offsetting. When considered i⿱⿱一口⿴囗十 terms of the arrangement of the normal mitral valvo, this is to be expected. The findings in the initial case were subsequently shown to be due to enlargement of the aortic root in the setting of Marfanos syndrome.

\section{Patients and heart studied}

The first patient was born at 34 weeks' gestatiog weighing $1.9 \mathrm{~kg}$ and was delivered by caesarean section because of maternal toxaemia of pregnanc\$ The early neonatal period was complicated by severe hyaline membrane disease and persistent fetal circu lation requiring intermittent positive ventilation for 10 days and tolazoline infusion for three days. After extubation the infant remained tachypnoeic. At seven weeks severe heart failure had developed and he was failing to thrive. During this time he was pir with normal peripheral pulses. There was leftwa迹 displacement of the cardiac apex and a promineat precordial impulse. A grade 4/6 pansystolic murmir 
and a grade $2 / 6$ mid-diastolic rumble were audible at the apex. He was also noted to have dysmorphic features with a high arched palate and elongated fingers. The electrocardiogram showed sinus rhythm with a normal precordial $R / S$ progression pattern for age. The chest radiograph showed a mild degree of pulmonary venous congestion with slight enlargement of heart size.

Cross sectional echocardiography showed a symmetrical arrangement of the inferior caval vein and abdominal aorta with the former to the right, indicating usual atrial arrangement (situs solitus). The usual offsetting of the atrioventricular valve was reversed in the apical four chamber section (fig 1), but the left sided ventricle was of a morphologically left pattern and the left valve was a typical mitral valve. There was moderate prolapse of both leaflets of the valve with enlargement of the left atrium. The

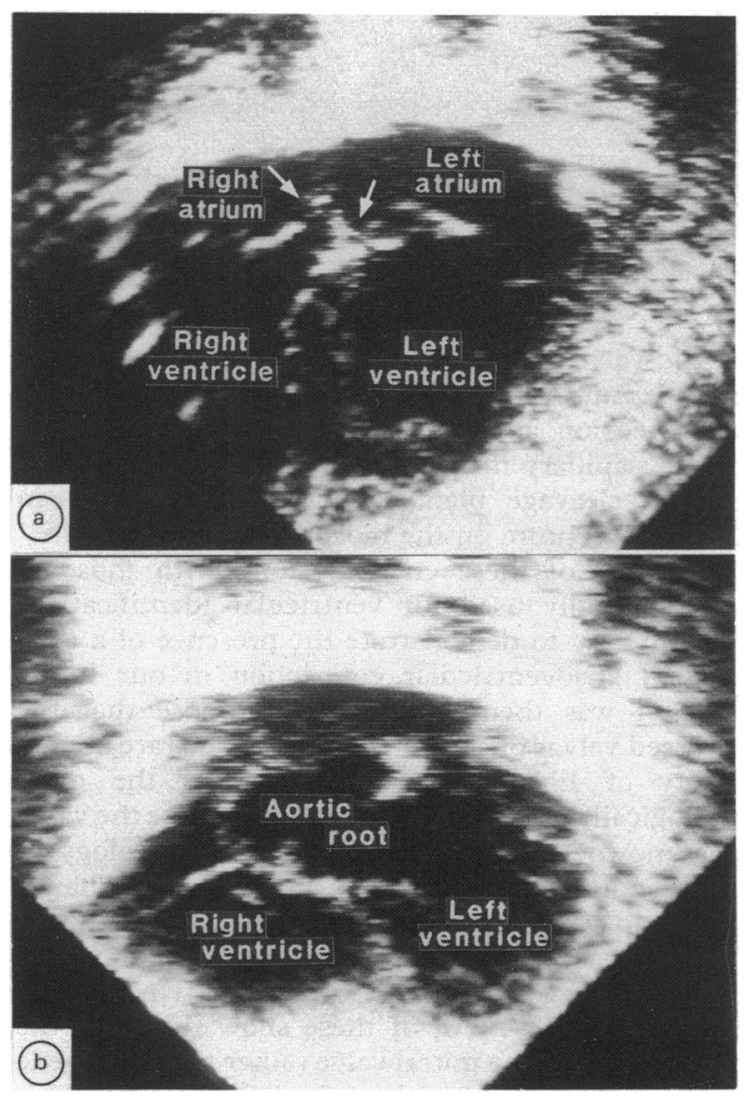

Fig 1 A four chamber echocardiogram (a) showing reversed offsetting of the septal attachments of the atrioventricular valves in a patient with usual atrial arrangement and concordant atrioventricular connection. The patient was also shown (b) to have gross dilatation of the aortic root. The diagnosis was Marfan's syndrome. ventriculoarterial connection was concordant and the great arteries were normally related. The aortic root was noted to be grossly dilated. Cardiac catheterisation excluded an intracardiac shunt and selective left ventriculograms showed mitral regurgitation and confirmed the presence of severe dilation of the aortic root. An initial diagnosis of Ebstein's anomaly of the mitral valve was considered because of the results of cross sectional echocardiography. This was not the case (see below). Instead, the reversed offsetting was the consequence of the severely dilated aortic root caused by Marfan's syndrome.

Subsequently we encountered a child who was shown during life to have Ebstein's malformation of the morphologically mitral valve. He presented with a heart murmur at six weeks of age and by four months became cyanosed when he cried and he was tachypnoeic. Cardiac catheterisation at the referring centre showed a normal atrial arrangement, a concordant atrioventricular connection, and double outlet right ventricle with a subaortic ventricular septal defect, pulmonary arterial and venous hypertension (mean left atrial pressure $30 \mathrm{~mm} \mathrm{Hg}$ ), and mitral regurgitation. The child was referred to the Hospital for Sick Children, Great Ormond Street, London at six months of age. Cross sectional echocardiogram demonstrated normal offsetting of the proximal septal attachments of the atrioventricular valves (fig 2a). The mural leaflet of the mitral valve, however, was grossly displaced towards the apex of the left ventricle (fig $2 \mathrm{~b}$ ). The mitral regurgitation, confirmed at catheterisation, was therefore a consequence of Ebstein's malformation of the morphologically mitral valve. The child was treated by banding of the pulmonary trunk and BlalockHanlon septectomy. This was successful and he was discharged home 10 days later and has done well for the past three years.

We had previously studied a heart from the Cardiopathological Museum of the Hospital for Sick Children, Great Ormond Street, London, which had been the arbiter of appropriate echocardiographic diagnosis during life. The atrial arrangement and chamber connections were all normal. The salient anatomy concerned the left atrioventricular valve. Although this was of a basically mitral pattern, the anatomy was more unusual. The aortic (anterior) leaflet of the valve was, as expected, in fibrous continuity with the aortic valve, but it showed a partial arcade lesion, the papillary muscles being continued as a muscular ridge along the leading edge of the valve leaflet. The mural aspect of the valve was grossly distorted, being densely adherent to the pariental wall of the ventricular inlet.with no true delamination of leaflet tissue (fig 3 ). There was 

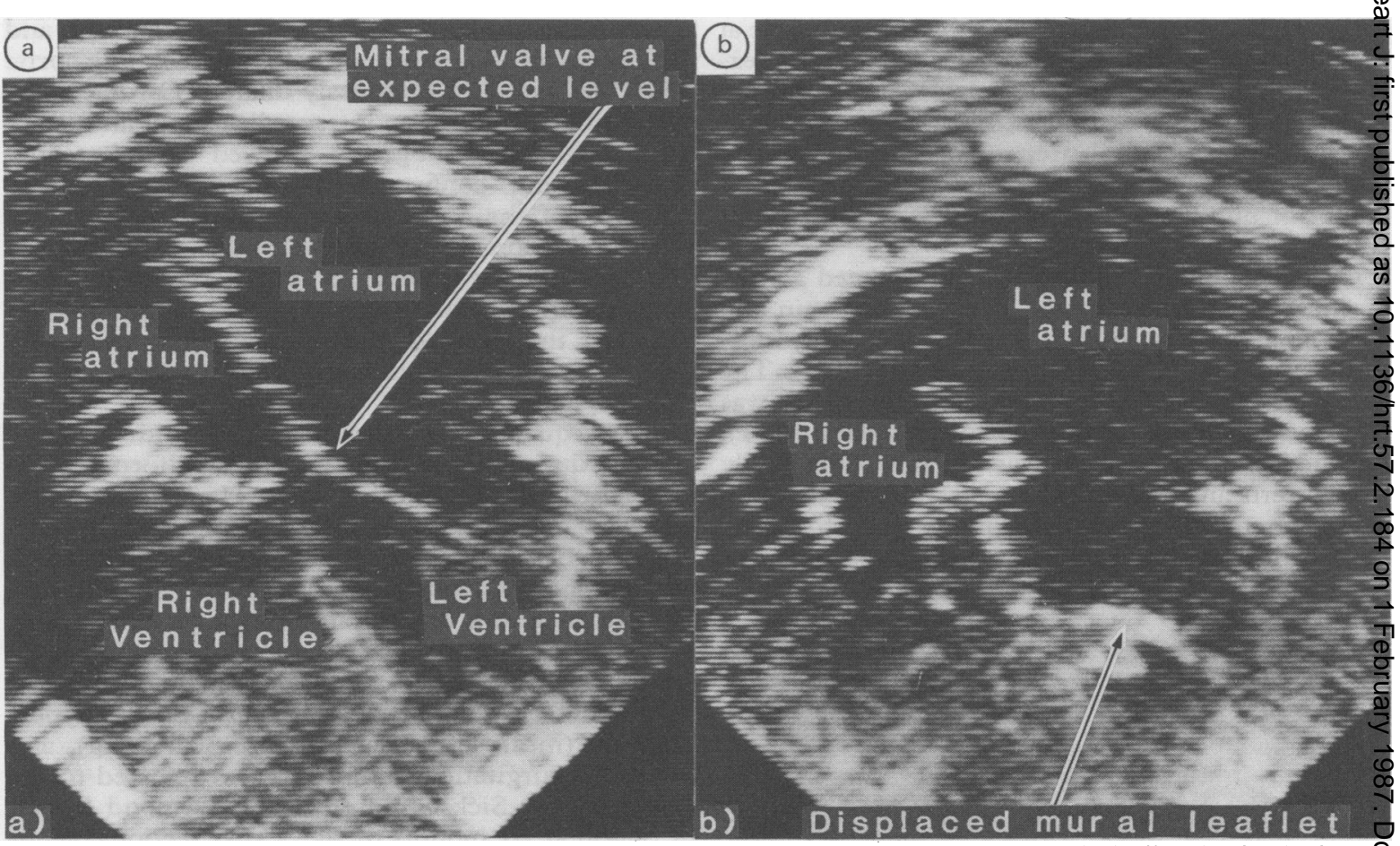

Fig 2 Cross sectional echocardiograms from a patient with Ebstein's malformation of the morphologically mitral valve showing (a) the normal offsetting of the atrioventricular valves and (b) the downward displacement of the mural leaflet.

no tension apparatus present for this part of the valve. The appearance was similar to that of Ebstein's anomaly of the morphologically tricuspid valve. In Ebstein's anomaly of the tricuspid valve, however, it is displacement of the septal leaflets that results in exaggerated offsetting of the annular attachment at the level of the atrioventricular septum. The mural rather than the septal leaflet of the mitral valve was found to be affected in this case. A four chamber section cut through the heart (fig 3b) showed that the aortic leaflet of the valve was attached at the expected position. The displaced segment of the valve was exclusively attached to the parietal wall of the ventricular inlet component. There was not, therefore, any reversal of the offsetting in the region of the atrioventricular septum.

\section{Discussion}

Reversed offsetting of the normal atrioventricular valve attachments in the setting of usual atrial arrangement (solitus) should alert the echocardiographer to the possibility of a discordant atrioventricular connection. A scan along the inlet ventricular septum will then demonstrate the attachment of the tension apparatus of the atrioventricular valves. The septal leaflet of the morphologically tri- cuspid valve always has multiple attachments to the inlet septum. This is in contrast with the mitrat valve tension apparatus which is attached to th $\overrightarrow{\vec{E}}$ paired papillary muscles away from the septum. I distinct cleavage plane is always found betwee valve and septum on the morphologically left side?

The use of these criteria, along with those of others usually used for ventricular identification made it easy to demonstrate the presence of a concordant atrioventricular connection in our initia case. It was then tempting to propose that thig reversed valvar offsetting was due to the rare abnot mality of Ebstein's malformation of the mog phologically mitral valve, particularly since the valve was known to be regurgitant. The morphology of this condition has been described by Ruschhaupt et $a l^{4}$ and we have seen both the case reported here (which has been illustrated elsewhere ${ }^{5}$ ) and a furthey case in the collection of Children's Hospital of Pittsburgh, USA. All of these show that it is the mural leaflet of the mitral valve rather than the aortif leaflet that is displaced into the left ventricle. This necropsy finding was subsequently confirmed in life by echocardiography of our second case. The arrangement is to be expected, since the aortic leaflet of the mitral valve, as its name suggests, is in fibrow continuity with the aortic valve. It spans the inled 


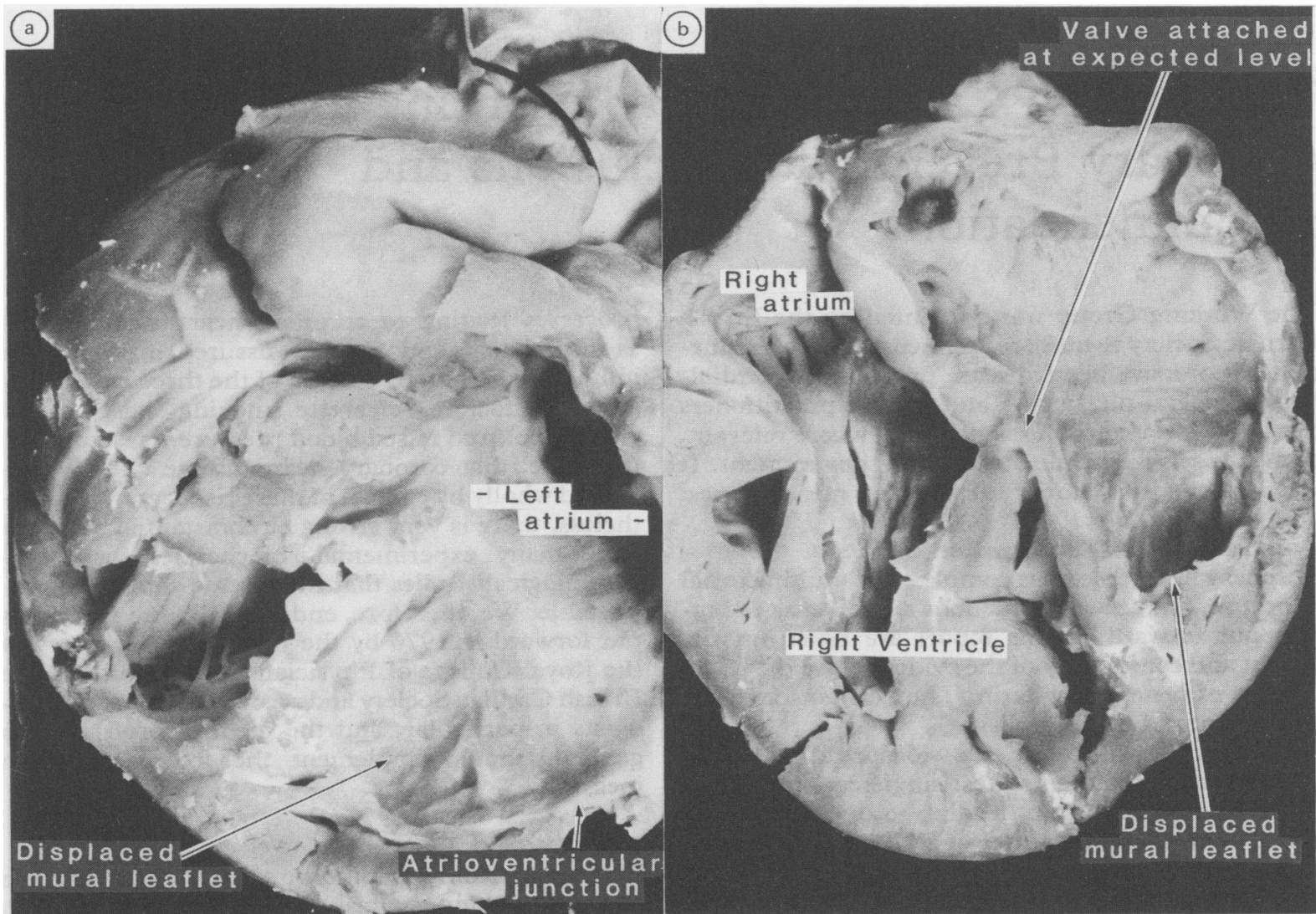

Fig 3 The salient morphological features of Ebstein's malformation of the morphologically mitral valve. (a) Shows the opened valve, the mural leaflet being downwardly displaced. The four chamber section (b) shows that the valve retains its expected level of attachment at the atrioventricular septum.

outlet junction of the left ventricle and cannot be displaced along the parietal wall or septum because it is not related to either of these structures. Therefore, apical displacement of the mitral valve leaflet was not the explanation for the echocardiographic features in our first patient. Rather it was upward displacement of the leaflets of the tricuspid valve by the dilated aortic root. The appropriate diagnosis is Marfan's syndrome. ${ }^{6}$ This is supported both by the dysmorphic features of the patient and the presence of prolapse of both leaflets of the mitral valve. In contrast, in the second case the mitral regurgitation was indeed due to Ebstein's malformation of the morphologically tricuspid valve. To the best of our knowledge this is the first non-invasive diagnosis of this rare lesion.

RHA is supported by the Joseph Levy Foundation and FJM by the Vandervell Foundation. They are both supported by the British Heart Foundation. ML was a visiting fellow at the Brompton Hospital from the Grantham Hospital, Hong Kong.

\section{References}

1 Foale R, Stefanini L, Rickards A, Somerville J. Left and right ventricular morphology in complex congenital heart disease defined by two dimensional echocardiography. Am f Cardiol 1982;49:93-9.

2 Tajik AJ, Seward JB, Hagler DJ, Mair DD, Lie JT. Two-dimensional real-time ultrasonic imaging of the heart and great vessels. Technique, image orientation, structure identification, and validation. Mayo Clin Proc 1978;53:271-303.

3 Sutherland GR, Smallhorn JF, Anderson RH, Rigby ML, Hunter S. Atrioventricular discordance: crosssectional echocardiographic-morphological correlative study. Br Heart f 1983;50:8-20.

4 Ruschhaupt DG, Bharati S, Lev M. Mitral valve malformation of Ebstein type in absence of corrected transposition. Am $\mathcal{F}$ Cardiol 1976;38:109-12.

5 Becker AE, Anderson RH. Pathology of congenital heart disease. London: Butterworths, 1981:149-51.

6 Sisk HE, Zakha KG, Pyeritz RE. The Marfan syndrome in early childhood: analysis of 15 patients diagnosed at less than 4 years of age. $\mathrm{Am} \mathcal{F}$ Cardiol 1983;52:353-8. 